\title{
Die Befruchtung und Entwicklung von Plattfischeiern in verdünntem Nordseewasser im Vergleich zu den Befunden in der freien Ostsee.
}

\author{
Von Wilhelm Marx und Johannes Henschel.
}

(Aus der Biologischen Anstalt auf Helgoland.)

(Mit 32 Abbildungen im Text.)

Inhaltsverzeichnis:

I. Vorbemerkung . . . . . . . . . . . . . . . . . . . . . . . . . . . . . 226

II. Fragestellung . . . . . . . . . . . . . . . . . . . . . . . . . . . . . . . . . 227

III. Die Versuche in verdünntem Nordseewasser: . . . . . . . . . . . . . . . . . . . . 228

1. Eier - und Larvenentwicklung in Seewasser mit verschiedenem Salzgehalt

a) Versuchsanordnung . . . . . . . . . . . . . . . . . . . . . . . . . . 228

b) Die Klieschenversuche . . . . . . . . . . . . . . . . . . . . . . . . . 230

c) Die Flunderversuche . . . . . . . . . . . . . . . . . . . . . . . . . . . 233

d) Besprechung der Ergebnisse: Die Brackwasserschädigung der Entwicklung . . . . . . 237

2. Sauerstoffzehrung von Kliescheneiern im Seewasser verschiedenen

Salzgehaltes . . . . . . . . . . . . . . . . . . . . . . . . . 237

a) Versuchsanordnung . . . . . . . . . . . . . . . . . . . . . . . . . . . . . 237

b) Versuchsergebnisse . . . . . . . . . . . . . . . . . . . . . . . . . . . . . . . . . 238

3. Die Befruchtungsrate vou Kliescheneiern im Seewasser verschiedenen

Salzgehaltes. . . . . . . . . . . . . . . . . . . . . . . . . . . . . . 239

IV. Vergleich zwisehen den Versuchsergebnissen und den Beobachtungen an Eiern und Larven in der freien Ostsee . . . . . . . . . . . . . . . . . . . . . . . . . . . . . . . 241

V. Zusammenfassung . . . . . . . . . . . . . . . . . . . . . . . . . . . . . . . 242

VI. Schriftenverzeichnis . . . . . . . . . . . . . . . . . . . . . . . . . . . . . . 243

\section{Vorbemerkung.}

Die vorliegende Abhandlung ist in der folgenden Weise zustande gekommen:

Der 1933 verstorbene Direktor der Biologischen Anstalt Prof. MtELck beabsichtigte nach seinen Beobachtungen bei den Fischbrutuntersuchungen in der Ostsee, die Fragen der Befruchtung der Fischeier und der Entwicklung der Larven durch Experimente klären zu lassen. Diese Versuche konnten jedoch erst einige Jahre spatter begonnen werden. Mit ihrer Durchführung wurde der damalige Studienassessor Dr. MaRx betraut, der schon 1931 und 1932 in seinen Ferien als wissenschaftliche Hilfskraft bei Prof. MTeLck tätig war und von Oktober 1934 bis Dezember 1935 als Forschungsassistent an der Biologischen Anstalt für die Austernzueht und für Fischbrutarbeiten angestellt war. Die Fischbrutversuche wurden von Dr. MARx persönlich von Dezember 1934 bis Februar 1935 durchgeführt.

Für die Versuche zur Feststellung der Sauerstoffzehrung der Kliescheneier stellte Dr. MaRX den Arbeitsplan und die Versnchsanordnung zusammen und ließ die Versuche selbst von dem Laboranten der Biologischen Anstalt, ANDREas HolTMAN durchfïhren, Ende März/April 1935, der ein sorgfältig aufgestelltes Protokoll ablieferte.

Alle Abbildungen der Arbeit wurden von Dr. MARx gezeichnet. Es war Dr. Marx nicht möglich, während seiner Helgolïnder Tätigkeit, die besonders stark durch die Austernzucht beansprucht war, die Ausarbeitung der Ergebnisse durchzuführen. Er wurde nach seinem Weggang von Helgoland im Schuldienst voll beschäftigt. Durch das Entgegenkommen seiner Behörde kounte Dr. MARX später in einem längeren 
Arbeitsurlaub einen zusammenfassenden Bericht ${ }^{\text {}}$ ) über die Fischbrutversuche und die künstliche Befruchtung der Kliescheneier ausarbeiten. Dieser Bericht mit den Abbildungen und die Aufzeichnungen von ANDREAS HOLTMANN über die Sauerstoffzehrung der Kliescheneier bildeten die Grundlage für die weitere Ausarbeitung der Abhandlung, mit der Anstalts-Assistent Dr. HENSCHEL vom Direktor der Biologischen Anstalt beauftragt wurde. Der Text wurde dazu im Einverständnis mit Dr MARX von Dr. Henschel neu verfaßt.

Es war durch die Versuche leider nicht möglich, die verschiedenen Fragen der Fischentwicklung in der Ostsee völlig zu klären. Sie geben jedoch verschiedene Hinweise, die bei einer weiteren experimentellen Verfolgung des Problems von Bedentung sein können. Daher erscheint die Veröffentlichung der Abhandlung durchaus gerechtfertigt.

\section{Fragestellung.}

Auf ihren wissenschaftlichen Forschungsfahrten beobachteten MIELCK und KüNNE (1935) im Plankton der Ostsee regelmäßig abgestorbene Fischeier. Diese Beobachtung war nicht neu. Schon APSTEIN (1911) bemerkte in seinem Untersuchungsmaterial aus der Ostsee zahlreiche tote Eier. Er bringt in seiner Arbeit Abbildungen davon. Die von ihm dargestellten Eier zeigen alle einen stark geschrumpften Inhalt (1911, S. 230). Es werden aber auch Eier beobachtet, die eine weiße Trübung des Dotters aufweisen, aber noch nicht geschrumpft, wahrscheinlich also eben gestorben waren. MiELcK glaubte, daß der größte Teil dieser toten Eier unbefruchtet gewesen ist, kann es aber nicht mit Sicherheit aussprechen. Wurden die noch gesund aussehenden Eier von ihm und KüNNE aussortiert und in Untersuchungsgläsern gehalten, traten nach kurzer Zeit ebenfalls Trübungen im Dotter zahlreicher noch lebender Eier auf, - hier allerdings auch an befruchteten Eiern - die bald darauf zu Boden sanken. Der Vorgang des Absterbens, den man an den verschiedenen Stadien geschrumpfter Eier für die freie Ostsee hatte ablesen können, setzte sich also in den Untersuchungsgläsern fort. Aehnliche Beobachtungen wurden an NordseeFischeiern nie gemacht. Im Nordseeplankton werden nur ganz vereinzelt tote Fischeier beobachtet.

Es liegt auf der Hand, für die große Sterblichkeit der Ostseeier hydrographische Faktoren verantwortlich zu machen und zunächst würde man hierbei an den Salzgehalt denken. Da gibt es nun verschiedene Möglichkeiten, in welcher Weise der Salzgehalt der Ostsee schädlich hätte einwirken können. Mielck erörtert diese Frage in der bereits zitierten Arbeit (S. 26-27). Er sieht in der Wasserschichtung der Ostsee die Hauptursache für die Schädigungen der Eier. Die Ostsee weist bekanntlich in ihren tiefen Becken eine salzreiche, kalte Tiefenschicht und eine salzarme, im Sommer wärmere Oberflächenschicht auf. Die Fische laichen dicht über dem Meeresboden in der Tiefenschicht, in der sich auch die Eier entwickeln. Beim Heraufholen mit dem Planktonnetz müssen sie einen jähen Salzgehaltswechsel erfahren, wenn sie in das salzarme Oberflächenwasser gelangen. Aber es ist seit den StrodtmanN'schen Untersuchungen (1906) bekannt, daß Fischeier eine recht große Salzgehaltsschwankung, und ebenso auch Temperaturschwankungen ertragen können. Immerhin konnte STRODTMANN einen stärkeren Ausfall an Eiern, die sich normal entwickelten, bei Salzgehaltserniedrigung beobachten.

Sodann wäre zu erwägen, ob nicht überhaupt der Salzgehalt auch der Tiefenschicht schon so gering ist, daß eine normale Befruchtung und Entwicklung nicht in allen Fällen zustande kommt. Besonders für die Befruchtung war dies seit den Beobachtungen Strodtmans's anzunehmen, der feststellte, daß Sperma vom Dorsch mit Salzgehaltserniedrigung die Beweglichkeit verliert (1906 S. 155). Die Wahrscheinlichkeit, daß ein schwebendes Ei befruchtet wird, müßte daher mit einer Abnahme des Salzgehaltes immer geringer werden. Die entweder bereits abgestorben heraufgeholten oder kurze Zeit darnach in den Versuchsgläsern eingegangenen Eier wären nach dieser Auffassung garnicht erst befruchtet worden. Und nach dem, was oben gesagt wurde, trifft dies für eine große Anzahl der Eier ja sicher zu.

Aber auch andere hydrographische Faktoren müssen als Ursachen der hohen Sterblichkeit der Ostseeier in Betracht gezogen werden. Die Bedeutung der Temperatur, besonders des Temperaturwechsels wurde bereits gestreift, der pH-Wert, der Sauerstoffgehalt, Nitrit- und Nitratgehalt sind weitere Eigenschaften, die auf Entwicklung und Widerstandsfähigkeit der Eier Einfluß乃 haben können. In der Arbeit MiELcK und KünNE (S. 37) wird weiter über die interessante Beobachtung berichtet, daß die Larven von Ostseefischen bei gleicher Länge auf einem früheren Entwicklungsstadium stehen als die Larven der Nordseefische. Dies Nachhinken der Entwicklung wird besonders deutlich an

1) Diese Arbeit wurde durch eine besondere Bewilligung von Mitteln seitens des Vorsitzenden der Deutschen wissenschaftlichen Kommission für Meeresforschung ermöglicht. 
der späteren Ausbildung der Flossenstrahlen. Beobachtet wurden diese Erscheinungen an der Flunder, Scholle, Hering, Ammodytes ${ }^{1}$ ), Cottus und wahrscheinlich auch an Dorsch und Sprott. Es werden Abbildungen von verschiedenen Larven aus Nord- und Ostsee gebracht (Tafel 2 Abb. 7-10 der erwähnten Arbeit). Da diese Abbildungen zur Zeit von MIELCK selbst veranlaßt und zusammengestellt wurden, ist anzunehmen, daß diese Beobachtung ihn veranlaßte, MARx zu den vorliegenden Versuchen anzuregen.

KünNE knüpft an diese Beobachtungen einige theoretische Betrachtungen. Manche Fischarten, z. B. der Dorsch und die im Bornholmbecken laichende Tiefenflunder, besitzen in der Ostsee größere Eier als in der Nordsee (KändLER $1934 \mathrm{~S} .57$, Strodtmann u. KändLer 1935 S. 295). Dies hängt zusammen mit der Anpassung der Eier an das spezifische Gewicht des verringerten Salzgehalts der Ostsee (STrodtmann 1906 S. 154). Aus diesén Eiern schlüpfen nun auch etwas größere Larven, die während ihrer Entwicklung den Größen-Vorsprung erhalten mögen. Vielleicht, meint. Künne, wäre hierauf die spätere Ausdifferenzierung der Gewebe zurürkzuführen. Dem widerspricht aber, daß auch die Larven diese Erscheinung zeigen, die in der Ostsee keine oder nur in sehr geringem Maße vergrößerte Eier besitzen, z. B. Hering und Scholle. Eine andere Erklärungsmöglichkeit sieht KünNe in einer biologischen Anpassung.

Die längeren, aber weniger weit entwickelten Larven müßten weniger Trockensubstanz besitzen als gleich lange Tiere, die schon weitere Organe angelegt haben. Dies müßte ihr spezifisches Gewicht verringern und könnte also das Schweben in dem brakkischen Ostseewasser erleichtern. KünNE spricht in diesem Zusammenhang von ökologischen und geographischen Rassen.

Ist aber nicht auch hier die naheliegendste Erklärung die des Salzgehaltseinflusses auf die Entwicklungsgeschwindigkeit? Eine ganz allgemeine Erscheinung der Tiere im Brackwasser ist die Größenreduktion (REmANe 1934 S. 62), die wahrscheinlich auf einer Verlangsamung der Lebensvorgänge beruht. Möglicherweise hat sich derselbe Salzgehaltseinfluß hier in einer etwas anderen Weise bemerkbar gemacht, etwa so, daß einfache Wachstumsvorgänge den komplizierten Differenzierungen vorweglaufen. Es kann in der Arbeit gezeigt werden, daß ein solches Nachhinken der Organbildung tatsächlich auch im Experiment beobachtet wurde.

\section{Die Versuche im verdünnten Nordseewasser.}

Bei den auf Helgoland ausgeführten Versuchen wurden verschiedene Probleme berührt. Zunächst wurde die Entwicklung von Eiern, die im normalen Salzgehalt befruchtet, darnach in verschieden verdünnte Salzgehaltsstufen überführt wurden, beobachtet. Eine andere Versuchsreihe befaßte sich mit der Befruchtungsrate in verschiedenen Salzgehaltsstufen. Ferner stand seit den Beobachtungen von Thienemann (1928), Schlieper (1930), BeADle (1931) das Interesse in Vordergrund, den Sauerstoff-Verbrauch in verschieden salzhaltigem Seewasser zu prüfen, da ja - wie bekannt - sich die Sauerstoff-Verbrauchswerte nach Umsetzen in verringerten Salzgehalt verändern. Es war daher auch für die vorliegende Studie wichtig, die Sauerstoffzehrung der Eier in verschiedenem Salzgehalt festzrstellen. Hiermit beschäftigte sich eine dritte Versuchsreihe.

\section{Eier- und Larvenentwicklung in Seewasser mit verschiedenem Salzgehalt.}

a) Versuchsanordnung.

Als Versuchstiere wurden Klieschen (Pleuronectes limanda) und Flundern (Pleuronectes flesus) benutzt. Die Eier wurden den Weibchen abgestreift und künstlich befruchtet. Die verschiedenen Salzgehalte ergaben sich aus entsprechenden Mischungen von Nordseewasser mit Regenwasser. Das vermischte Wasser wurde durch Hydraffinkohle filtriert.

Als Versuchsgefäße dienten für die Klieschenuntersuchungen Aquarien von 31 Inhalt, die im Physiologischen Zimmer des Hafenlaboratoriums standen. 'Das Wasser wurde im ersten Versuch alle drei Tage gewechselt. Es war dafür gesorgt, daß die Temperatur annähernd konstant blieb. Von normalem Salzgehalt bis zu der Salzgehaltsstufe

1) I)r. KändLER, Kitzeberg teilte uns freundlicherweise mit, daß3 er dieselbe Beobachtung an den Larven von Ammodytes marinus gemacht hat, während die Larven von A. tobianus sich in Nord- und Ostsee gleichen. 
von $18 \%$ verlief die Entwicklung normal. Daher sind für die Auswertung nur die Ergebnisse der Kontrollzucht aus normalem Seewasser und die der Zuchten aus 18\% und niedriger berücksichtigt.

Für die Flunderversuche wurde eine andere Versuchsanordnung gewählt. Das Wasser, das, wie gesagt, alle drei Tage gewechselt wurde, muß sich im Laufe dieser dreitägigen Periode in Bezug auf verschiedene Wasserfaktoren geändert haben. Der Sauerstoffgehalt muß durch Sauerstoffzehrung am Ende jeder Periode geringer geworden sein, der $\mathrm{pH}$-Wert muß sich geändert haben, Nitrit- und Nitratgehalt müssen gestiegen sein. Dies alles sind Faktoren, die die Entwicklung möglicherweise beeinflußt haben. Um solche Versuchsfehler auszuschalten, wurden die Flundereier im fließenden Wasser gehalten. Dafür wurde das Versuchsbecken (Abb. 1) zwischen zwei Wasserbehälter geschaltet.

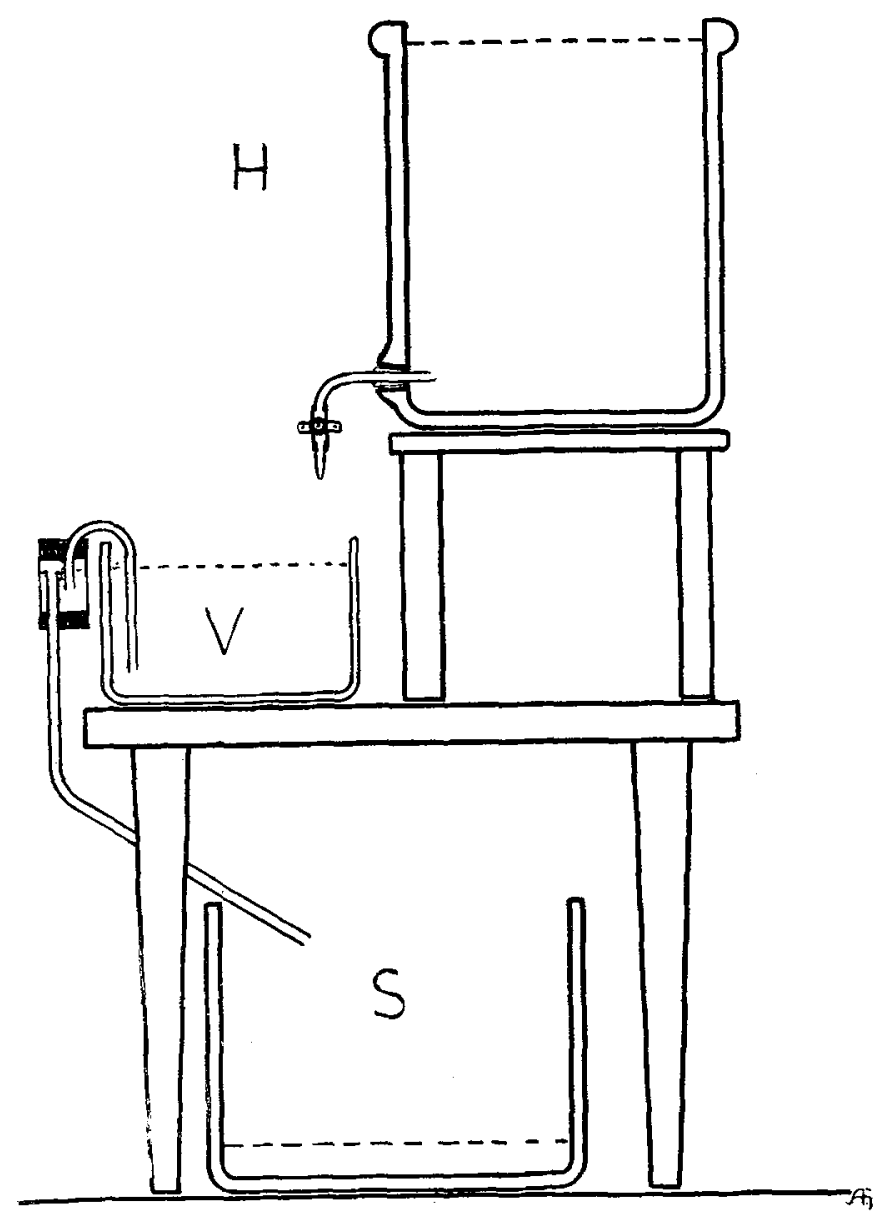

Abb, 1. Versuchsanorduung für künstliche Aufzucht von Flundereiern.

Erklärung; $H=$ Hochbehïlter, $V=$ Versuchsgefä $\beta, S=$ Sammelbecken.

Aus dem oberen Becken floß Wasser durch eine Rohrleitung mit Regulierhahn ins Versuchsbecken. Aus diesem heberte es sich durch eine Hebervorrichtung selbsttätig ab. Das verbrauchte Wasser wurde in einem tiefer stehenden Becken aufgefangen, konnte darnach wieder filtriert und durch starke Durchlüftung mit Sauerstoff neu gesättigt werden, sodaß es sich vermeiden lief, dauernd neue Seewassermischungen vorzunehmen. Natürlich wurde das Seewasser mit normalem Salzgehalt für die Kontrollversuche genau so behandelt. Der Wasserzufluß war so reguliert, daß in jeder Stunde 1 Liter Wasser zu- und abfloß. Die Versuchsbecken dieser Versuchsreihe enthielten je $4 \mathrm{l}$, somit wurde das gesamte Wasser 6 mal am Tage erneuert. pH-Wert und Sauerstoffgehalt wurden dauernd überprüft. Die Messungen bewiesen, daß diese Faktoren im Gleichgewicht blieben. 


\section{b) Die Klieschenversuche.}

Zwei Versuchsreihen wurden mit Klieschen ausgeführt. Beim ersten Versuch wurden die Eier einen Tag nach der künstlichen Befruchtung, nachdem die unbefruchteten Eier schon gestorben und zu Boden gesunken waren, in die Salzgehaltsstufen von $32,3 \%$, $18,4 \%, 15,2 \%, 11,2 \%$ und $8,2 \%$ überführt. Die Temperatur schwankte im Laufe der Entwicklungszeit zwischen 9 und $11^{\circ} \mathrm{C}$. Die Entwicklung dauerte 5-7 Tage, nach welcher Zeit die Larven, die sich normal entwickelt hatten, ausgeschlüpft waren.

Der niedrige Salzgehalt hat in verschiedener Weise auf die Entwicklung der Eier eingewirkt. Zunäehst, bereits vom 2. Tage an nach Umsetzen in den verringerten Salzgehalt, traten Quellungen des Dotters auf. Diese wurden an Volumenvergrößerungen des Dottersacks erkannt, wodurch der perivitelline Raum mehr und mehr verkleinert wurde. Die Abbildungen $2-6^{1}$ ) zeigen gleichaltrige Eier aus den 5 Salzgehaltsstufen. Man bemerkt

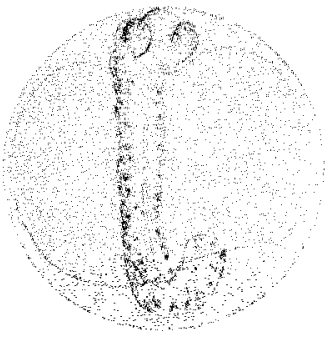

Abb. 2. Klieschenei aus normalem Nordseewasser, 3 Tage nach Befruchtung.

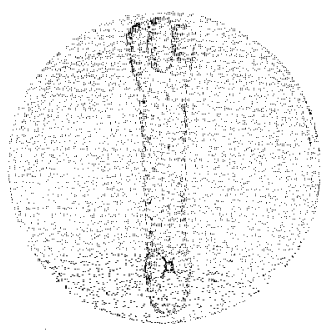

Abb. 3. Klieschenei, 3 Tage nach Befruchtung; die letzten 2 Tage in verdünntem Nordseewasser von $18,4 \%$.

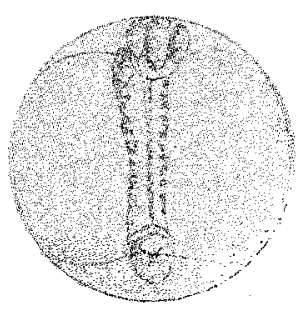

Abb. 4. Klieschenei, 3 Tage nach Befruchtung; die letzten 2 Tage in verdünntem Nordseewasser von $15,2 \%$

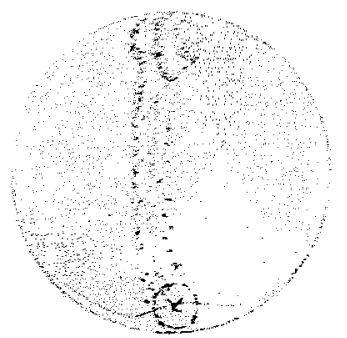

Abb. 5. Klieschenei, 3 Tage nach Befruchtung; die letzten 2 Tage in verdünntem Nordseewasser von $11,2^{\circ}{ }^{\prime}{ }^{\prime}$.

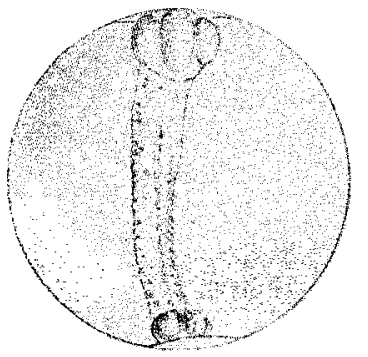

Abb. 6. Klieschenei, $\delta$ Tage nach Befruchtung; die letzten 2 Tage in verdünntem Nordseewasser von $8,2 \%$.

eine Zunahme der Quellung von 18,4 bis $8,2 \%$. Im Salzgehalt von $8,2 \%$ ist der perivitelline Raum vollkommen verschwunden.

Eine weitere Wirkung bestand in einer Entwicklungshemmung des Embryos, die besonders deutlich in der späteren Ausbildung der Augen und des Pigments in Erscheinung trat und sich schließlich in einer zunehmenden Verschiebung der Schlüpfzeit bemerkbar machte, je geringer der Salzgehalt war. Aus den Aufzeichnungen geht folgende Aufstellung hervor:

Ausschlüpfen der Larven vom 6. bis 12. Tage nach Versuchsbeginn:

$\begin{array}{lccccc}\text { In Salzgehalt: } & 32,3 \% & 18,4 \% & 15,2 \% & 11,2 \% & 8,2 \% \\ \text { Am 6. Tage: } & \text { alle } & \text { einige } & \text { keine } & \text { keine } & \text { keine } \\ \text { Am 7. Tage: } & - & \text { die Mehrzahl } & \text { ein Teil } & \text { keine } & \text { keine } \\ \text { Pause v. 8. -11. Tage } & & - & - & \text { einige } & \text { keine } \\ \text { Am 12. Tage: } & - & - & - & \end{array}$

In $8,2 \%$ schlüpfen also überhaupt keine Larven, in 15,2 und $11,2 \%$ schlüpfte nur ein Teil von ihnen. Durch den gequollenen Dotter wurden die Larven vielleicht behindert, sich aus der Eihülle zu zwängen. Sie blieben größtenteils darinnen stecken

1) Die Abbildungen 2-31 wurden von MARx mit dem Zeichenapparat nach dem lebenden Objekt gezeichnet. (Vergrößßerung 30 fach.) 
und gingen zugrunde. Fs ist aber noch eine andere Erklärung möglich. In den niedrigen Salzgehaltsstufen platzten bereits 5 Tage vor dem Schlüpfen bezw. vor den miBglückten Schlüpfungsversuchen die Eihüllen. Die Zuchten waren stark verpilzt. Diese Erscheinungen erimnern an Beobachtungen von Henschel, über die v. Bundensrock berichtet (s. Reibisch 1931 S. 250). Darnach werden die Eihüllen der Schollen- und Flundereier durch Verpilzung vorzeitig zerstört. Die Larven geraten vor Abschluß ihrer Embryonalentwicklung mit dem Außenmedium in Berührung, wodurch sie nach einiger Zeit zugrunde gehen. Die aus den Eihüllen heraushängenden Larven der niedrigen Salzgehaltsstufen könnten solche vorzeitig mit dem Aubenmedium in Berührung geratenen Larven sein, die sich in unverpilztem Zustand noch weiter entwickelt hätten und später möglicherweise normal geschlüplit wären.

In den niedrigen Salzgehaltsstufen wurden schließlich Mißßbildungen beobachtet, besonders stark in 11,2 und $8.2 "$ " die mit dem Wachstum zunahmen. Auch Verkümmerungen der Flossensäume gehören zu den pathologischen Erscheinungen.

In einem zweiten Versuch wurden die Eier erst nach Bildung des Embryos aus normalem Nordseewasser in die entsprechenden Verdünnungsstufen überführt, zu einem Zeitpunkt, an dem die Banchdecken des Embryos den Dotersack bereits umwachsen hatten. Es sollte festgestellt werden, ob die ausgebildete Haut dem Tier einen osmotischen Schutz bietet und eine Quellung des Dotters verhindert. In einer ersten Versuchsreihe wurden die Tiere drei Tage nach Befruchting in die bekannten Salzgehaltsstufen 18,4, 15,2, 11,2 und $8,2^{\prime \prime}$, überführt. Die Temperatur betrug in diesem Versueh nur 8-9" C, die Entwicklung dauerte entsprechend länger, die ersten Larven schlüpften am 9. Tage. Wenn auch die Schliipfergebnisse in dieser Versuchsreihe etwas besser gewesen sind als im 1. Versuch - es kamen z. B. in 8,2" $2^{\circ}$ auch einige Larven zum schlüpfen, - so traten doch auch hier die typischen Erscheinungen in den geringen Salzgehalten wieder auf: Quellung des Dotters, Verlangsamung der Entwicklung und auch Mißbildung. Dies zeigen einige Bilder. Die Eier der Abbildungen 7 und 8 sind 7 Tare alt gewesen. Abbildung 8 bringt

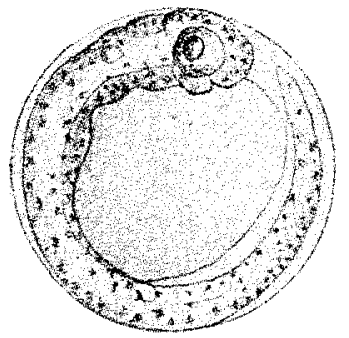

Abb. 7. Klieschenei, T Tare nach Befruchtung, aus normalem Nordseewasser.

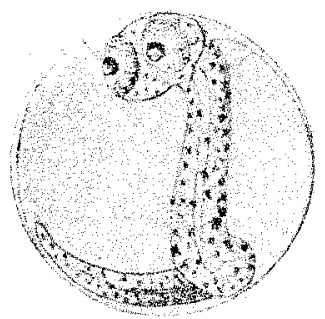

Abb. 8. Klieschenei, 7 Tage nach Befruchtung. 1) Ei befand sich seit den 4 Entwicklungstag in verdüntem Nordseewasser von $8,2 \%$.

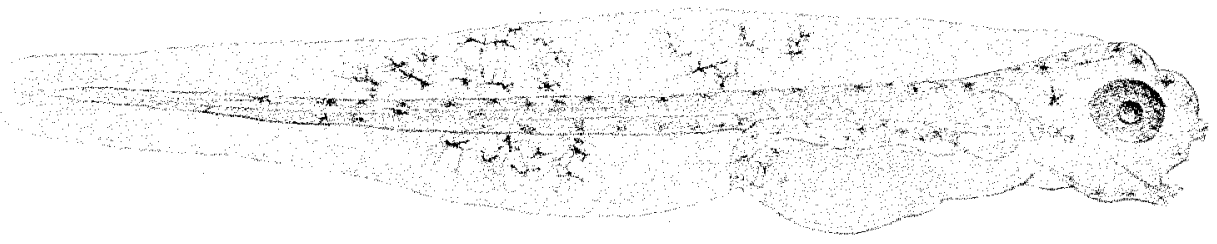

Abb. 9. Klieschenlare aus normalem Nordseewasser. frisch geschliupt.

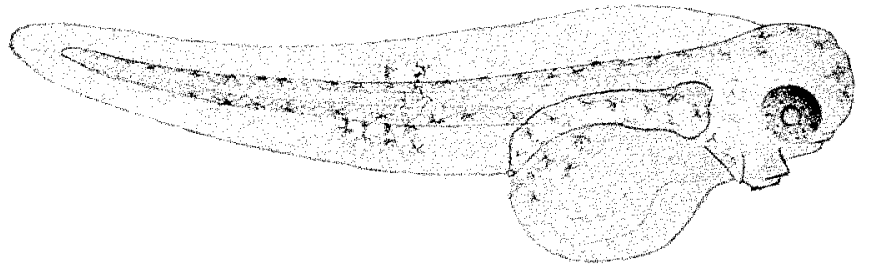

Abb. 10. Klieschenlarve frisch geschlüpft. Das Ei befand sich seit dem 4. Eutwicklungstag in verdünntem Nordseewasser von $11,2 \%$. 


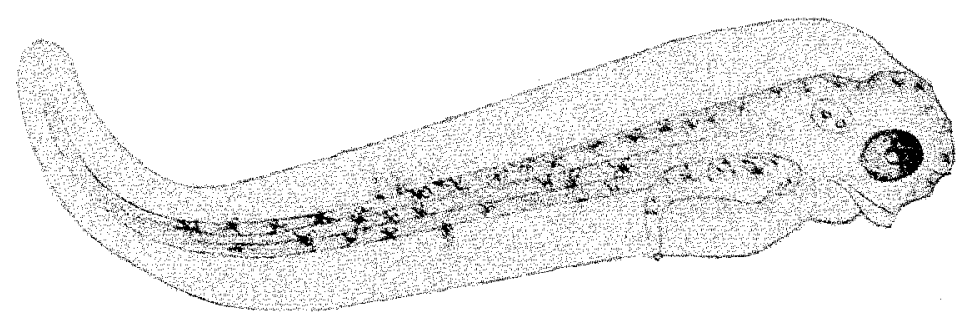

Abb. 11. Klieschenlarve, frisch geschlüpft. Eientwicklung zunächst in normalem, nur am letzten Tag vor dem Schlüpfen in verdünntem Nordseewasser ron $15,2^{\circ}, 00$.

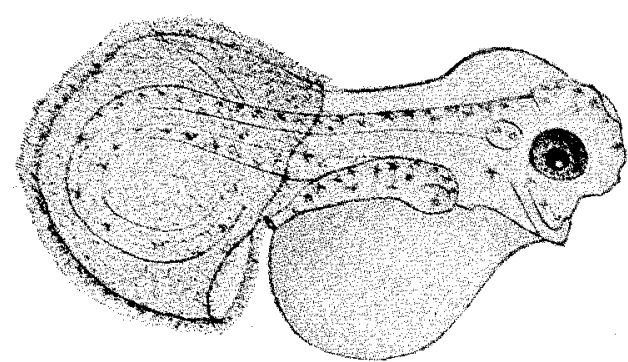

Abb. 12. Schlüpfende Klieschenlarve (Eihaut rerpizt). Fientwicklung zunächst in normalem, aur am letzten letzten Tag ror dem Schlüfeu in verdünntem Nordseewasser von $11,2 \%$ \%

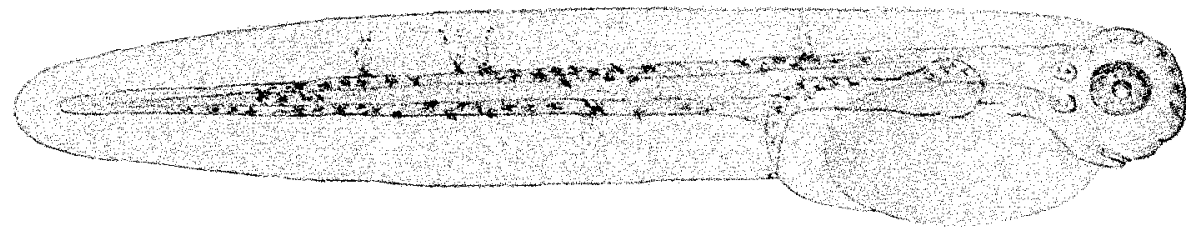

Abb. 13. Klieschenlarve, 3 Taye nach dem Schlüpfen, aus normalem Nordseewasser.

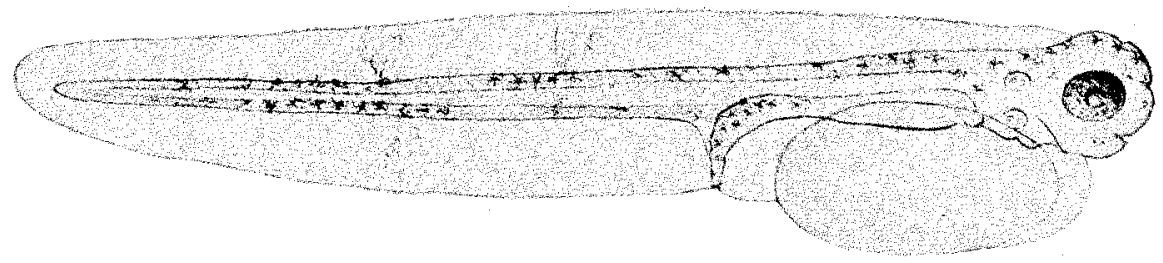

Abb. 14. Klieschenlare, 3 Tage nach dem Schlïpten, sofort nath dem Schliplen in verdinntes Nordseewasser von 11,2 " äberführt.

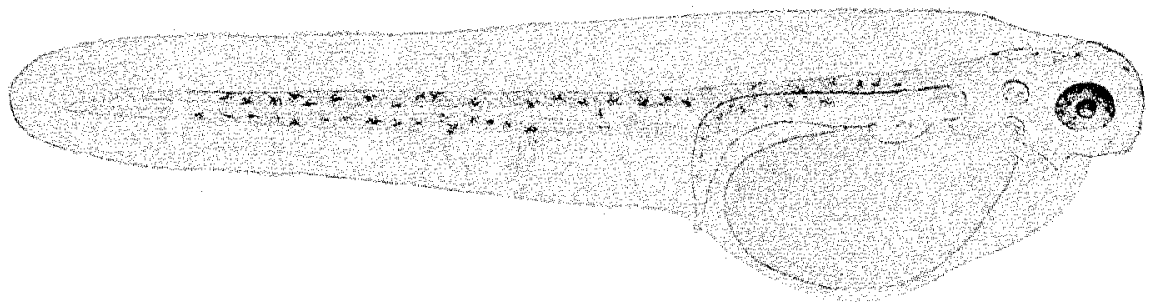

Abb. 15. Klieschenlarve, 3 Tage nach dem Schlüpfen, sofort nach dem Schlüpfen in verdünntes Nordseewasser von $8,2 \%$ überführt. 


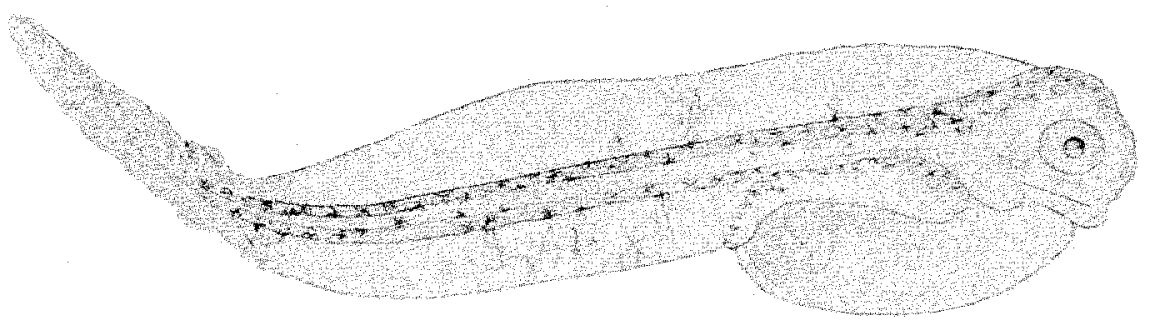

Abb. 16. Klieschenlarve wie aus Abb. 15 mit zerstörtem Schwanzende.

die Zeichnung eines Eies aus 32,3\%, Abb. 9 aus 8,2\% dem Einfluß des niedrigen Salzgehalts ausgesetzt. Die Quellung des Dotters fällt wieder deutlich ins Auge. Der perivitelline Raum ist sehr verkleinert worden. Weiter zeigt die Larve eine Krümmung, die der normalen Lage nicht entspricht.

Abb. 9 und 10 geben die Bilder von zwei geschlüpften Larven aus 32,3 und $11,2^{\prime \prime} / 00$. Man erkennt auch an ihnen wieder die schädlichen Einflüsse des geringen Salzgehalts. Die Brackwasserlarve ist kleiner, ihr Dottersack weniger stark resorbiert, er reicht noch bis in die Region des Unterkiefers. Weniger differenziert als in normalem Seewasser ist auch die Kopfregion. Die Brustflosse ist überhaupt noch nicht entwickelt, das Pigment ist spärlicher ausgebildet. Eine geringe dorsale Krümmung des Rumpfes und des Schwanzendes und die Verkümmerung der Flossensäume deuten pathologische Bildungen an.

In einer weiteren Selie wurden die Tiere erst 5 Tage nach Befruchtung in die Salzgehaltsverdünnungen überführt. Bei der hohen Wassertemperatur, die während der Dauer des zweiten Versuchs herrschte $\left(10^{\circ} \mathrm{C}\right)$, waren sie bereits sehr weit entwickelt. Sie schlüpften einen Tag später, am 6. Tage also. Sogar an diesen Tieren zeigten sich in 15,2 und 11,2\% die bekannten Schädigungen (Abb. 11 und 12), Von Eiern aus 8,2\% liegen keine Zeichnungen vor.

Die Ergebnisse des 2. Versuchs beweisen, daß die ausgebildete Körperhaut den Larven keinen osmotischen Schutz bietet. Larven, die nach dem Schlüpfen in die verdünnten Salzgehalte überführt wurden, zeigten ebenfalls Schädigungen: Quellungen des noch nicht vollständig resorbierten Dottersacks, geringe Verzögerungen der Entwicklung, die besonders deutlich wird an der spärlicheren Ausbildung des Pigments (Abb. 13, 14 und 15 ). Später wurden auch Mißbildungen beobachtet, Verkrümmungen und Zerstörungen der Gewebe besonders in der Schwanzregion, wie es die Abb. 16 zeigt.

\section{c) Die Flunderversuche.}

Im Anschluß an die Versuche mit Kliescheneiern wurden auch Flunderversuche ausgeführt, bei denen die oben beschriebene (Seite 229), verbesserte Versuchsanordnung verwendet wurde. Dieselben Mischungsverhältnisse des Nordseewassers mit Regenwasser wie im Klieschenversuch ergaben annähernd gleiche Konzentrationsstufen. Nur die geringste Salzgehaltsstufe wich etwas stärker von der entsprechenden des Klieschenversuchs ab; sie betrug $7,3 \%$. So ergeben sich folgende Salzgehalte, in denen Flunrereier gehalten wurden: $32,3 \%, 18,2 \%, 14,6 \%, 11,3 \%$ und $7,3 \%$. Die Temperatur betrug am Anfang des Versuchs $4^{\circ} \mathrm{C}$, stieg gegen Ende bis $7^{\circ} \mathrm{C}$ an. Der Anstieg vollzog sich aber in allen Salzgehaltsstufen gleichmäBig. Die Eier wurden im 4-Zellenstadium in die verschiedenen Salzgehalte verteilt. Die ersten Larven schlïpften am 8. Tage nach der Befruchtung. Die Eier haben also recht lange unter der Einwirkung des schwachsalzhaltigen Wassers gestanden.

Während sich in den ersten drei Tagen die Eier in allen Versuchsbecken gleichmäBig entwickelten, konnte man am 4. Tage nach Befruchtung eine Verengung des perivitellinen Raumes, also eine Dotterquellung, in 11,3 und $7,3 \%$ beobachten. Die Wirkungen der niedrigen Seewasserkonzentrationen auf diese frühen Stadien der Entwicklung zeigt die Bildserie der Abbildungen 17, 18 und 19. Besonders am Ei aus 7,3\% ist die Quellung des Dotters gut sichtbar. Der Embryo zeigt weniger scharfe Umrisse als im normalen Salzgehalt, die Seitenränder des Rumpfes erscheinen nicht gerade gezogen, sie sehen eher etwas gewellt und ausgefranst aus, man hat den Eindruck, daß die osmotischen Schädigungen bereits das Gewebe des Embryo in Mitleidenschaft gezogen haben. Bei dem Embryo aus dem Seewasser von 7,3\% ist die spärlichere Entwicklung des Pigments augenfällig, ebenso auffallend die weniger weite Ausbildung der Augen. 


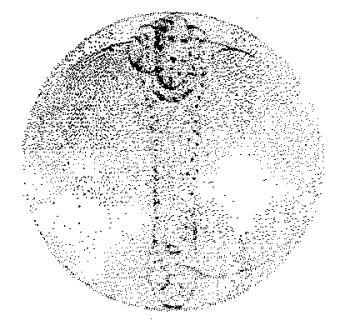

Abb. 17. Flunderei aus normalem Nordseewasser, 3 Tage nach Befruchting.

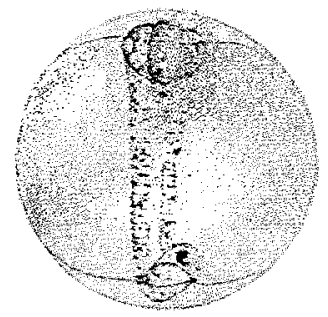

Abb. 18. Flunderei, 3 Tage nach Befruchtung, das im 4-Zellenstadium in verdüntes Nordseewasser von $11,3 \%$ iiberführt wurde.

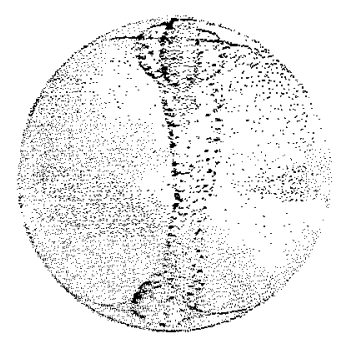

Abb. 19. Flunderei, 3 Tage nach Befruchtung, das im 4-Zellenstadium in verdünntes Nordseewasser von $7,3 \%$ uberführt wurde.

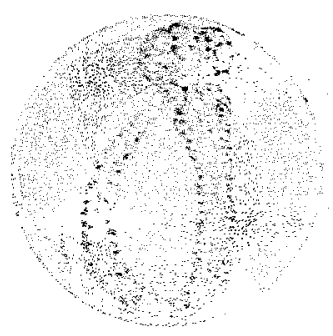

Abb. 20. Flunderei, 7 Tage nach Befuchtung, aus normalem Nordseewasser.

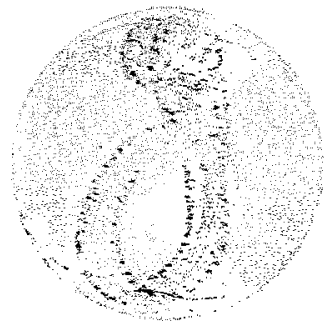

Abb. 21. Flunderei, $\rightarrow$ Tage nach Befruchtung, das im 4-Zellenstadium in verdïnntes Nordseewasser von 11,3 "ino überfinint wurde.

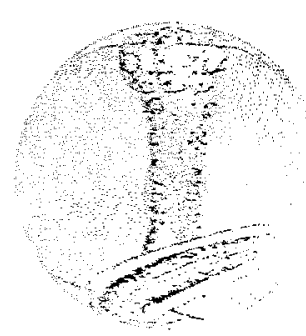

Abb. 22. Flunderei, 7 Tage nach Befruchtung, das im 4-Zellenstadium in verdünntes Nordseewasser von $7,3 \%$ überführt wurde.

Drei Tage später, also 7 Tage nach der Befruchtung, traten Biegungen und Verkrümmungen des Embryos auf, besonders stark an Eiern aus 7,2\% (Abb. 20-22). Rumpf- und Schwanzanlage sind weniger differenziert, die Flossensäume der Brackwasserindividuen geringer entwickelt.

Noch deutlicher treten die Einwirkungen des geringen Salzgehalts an den geschlüpften Larven in Erscheinung (Abb. 23, 24 und 25). Geringe Pigmententwicklung, Verkümmerung des dorsalen Flossensaumes an der 7,3\% -Larve, schließlich ein größerer Dottersack, der wohl auf Quellungen des Dotters deutet. Auch in diesen Bildern erscheinen die Umrisse der Brackwasserlarven, besonders wieder die der Larven aus $7,3 \%$, gewellt und unschärfer als die Umrisse der Larven aus normalem Seewasser.

Die Entwicklungshemmungen setzen sich in der Folgezeit, während welcher die Larve den Dottersack resorbiert, fort. Dies zeigen die beiden folgenden Bilderreihen: Abb. 26, 27 und 28 zeigt Larven des 12., Abb. 29, 30 und 31 solche des 14. Entwicklungstages. Die Pigmententwicklung bleibt auch bei den Larven weiter zurück; der Kopf erscheint wie im Klieschenversuch weniger differenziert, - man beachte besonders die Form des Unterkiefers - in 7,3\% bilden sich schließlich Krüppelformen aus, vollkommen verkrümmte, nicht lebensfähige Larven, wie Abb. 31 eine solche zeigt.

Auch in diesem Flunderversuch verschob sich die Zeit des Schlüpfens mit zunehmender Salzgehaltsverdünnung. Dafür sei hier eine Beobachtungsreihe gebracht, wie sie aus den Aufzeichnungen der Versuchsprotokolle hervorgeht.

Ausschlüpfen der Larven vom 8. bis 12. Tage nach Versuchsbeginn.

Im Salzgehalt:

Am 8. Tage:

Am 9. Tage:

Am 10. Tage:

Am 11. Tage keine Aufzeichnungen.

Am 12. Tage:

$$
\begin{array}{cc}
32,3 \% & 18,2^{\circ} \% \\
\text { wenig } & \text { wenig } \\
\text { alle } & \text { fast alle } \\
- & \text { der Rest }
\end{array}
$$$$
\text { ufze }
$$

$$
\begin{gathered}
14,6 \% 00 \\
\text { wenig } \\
\text { ein Teil } \\
\text { ein Teil }
\end{gathered}
$$

die letzten

$$
\begin{array}{cc}
11,3 \% & 7,3^{0} / 00 \\
\text { wenig } & \text { wenig } \\
\text { ein kl. Teil } & \text { ein kl. Teil } \\
\text { ein Teil } & \text { ein Teil }
\end{array}
$$

etwa $1 / 2 \quad$ etwa $1 / 4$




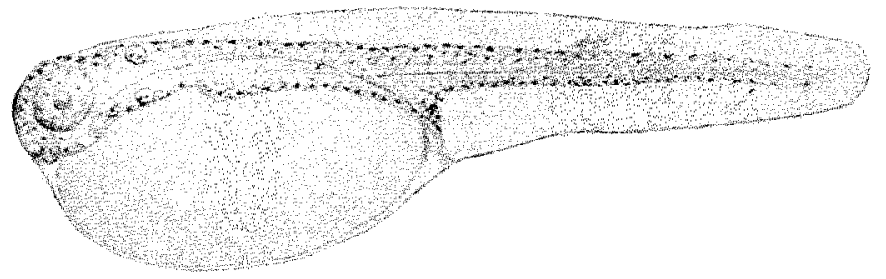

Abb. 23. Fluuderlarve aus normalem Noriseewasser.

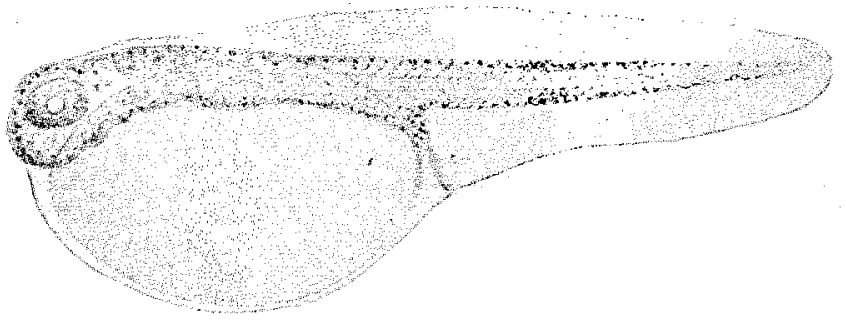

Abb. 24. Flunderlarve aus rerdünntem Nordseewasser von $11,3^{\circ}{ }^{\prime} 0^{\circ}$

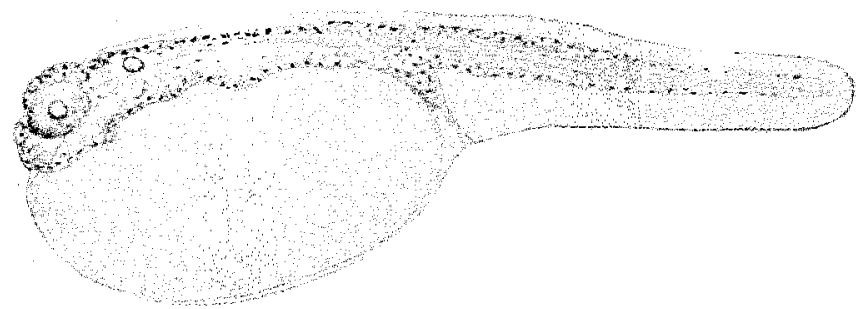

Abb. 25. Flunderlarve aus verdinntem Nordseewasser von $7,3 \%$.

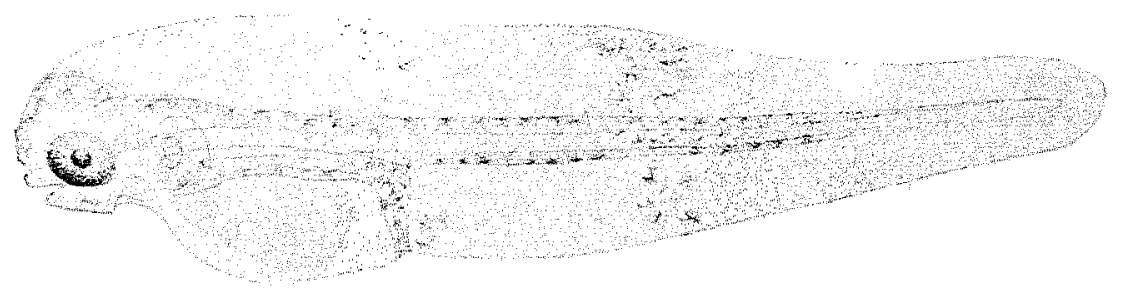

Abb. 26. Flunderlarve am 12. Tag nach Befruchtung des Eies, aus normalem Nordseewasser.

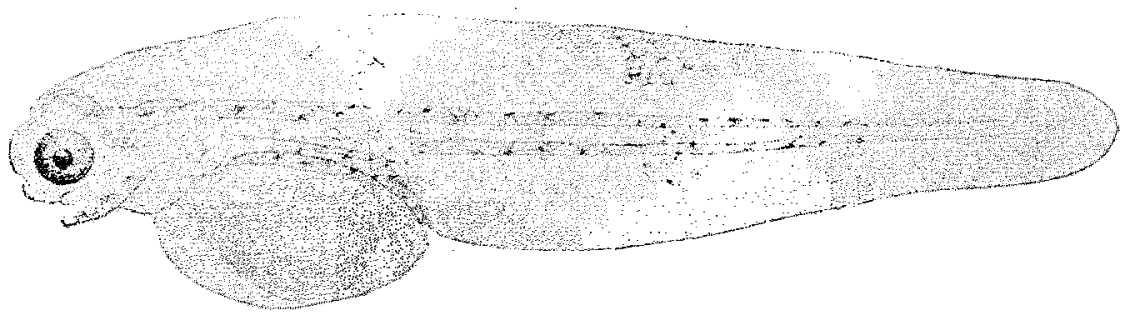

Abb. 27. Flunderlarve am 12. Tag nach Befruchtung des Eies, aus verdünntem Nordseewasser von $11,3 \%$ o . 


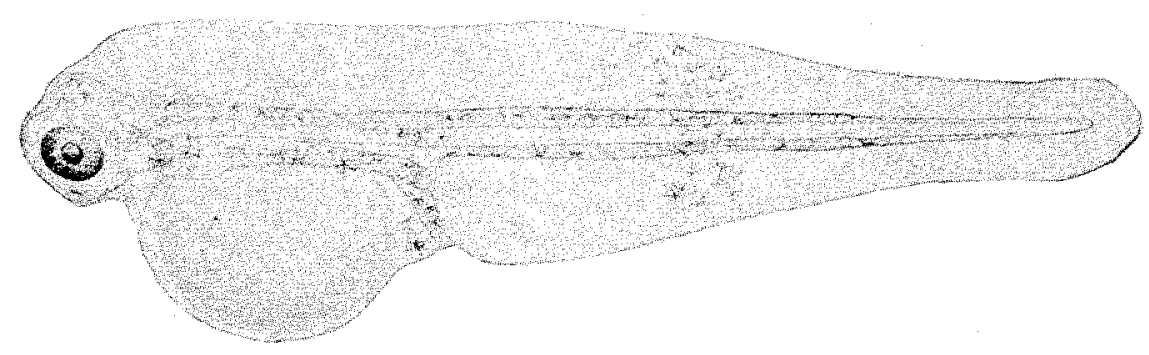

Abb. 28. Flunderlare am 13. Tag nach Befruchtung des Eies, aus verdïntem Nordseewasser von $7,33^{\circ}$ im.

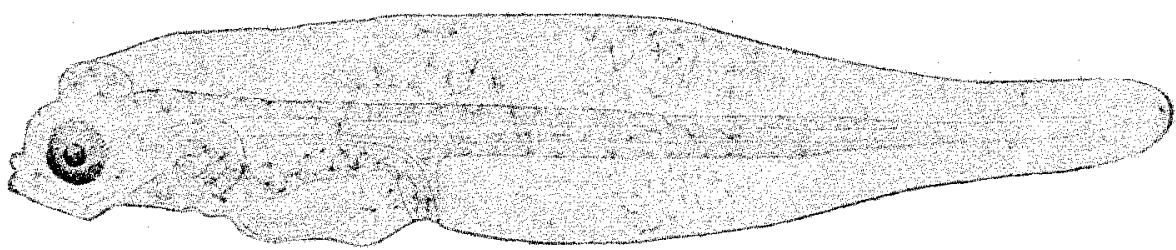

Abb. 29. Flanderlarve an 14. Tag nach Beiruchtung des Eies, alls nomalen Nordseewasser.

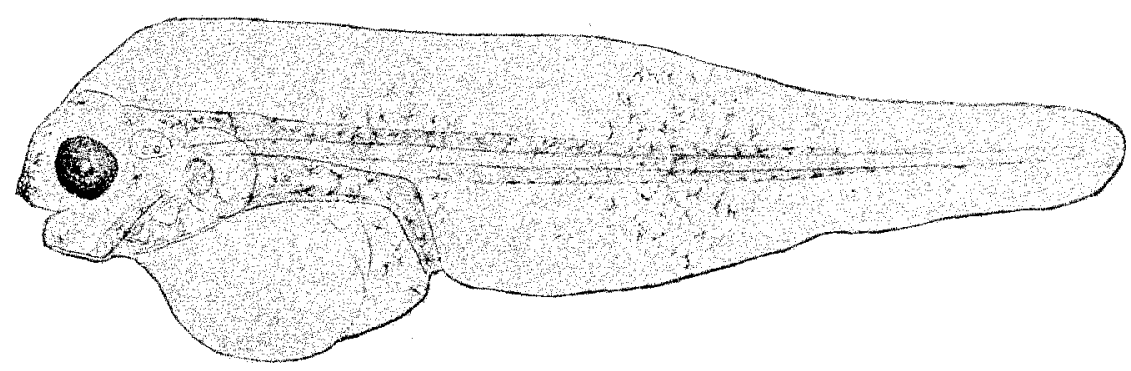

Abb. 30. Flunderlarve an 14. Tag narh Befruchtung des Eies, aus verdïnntem Norrseewasser von $11,3^{\prime \prime}$,0.

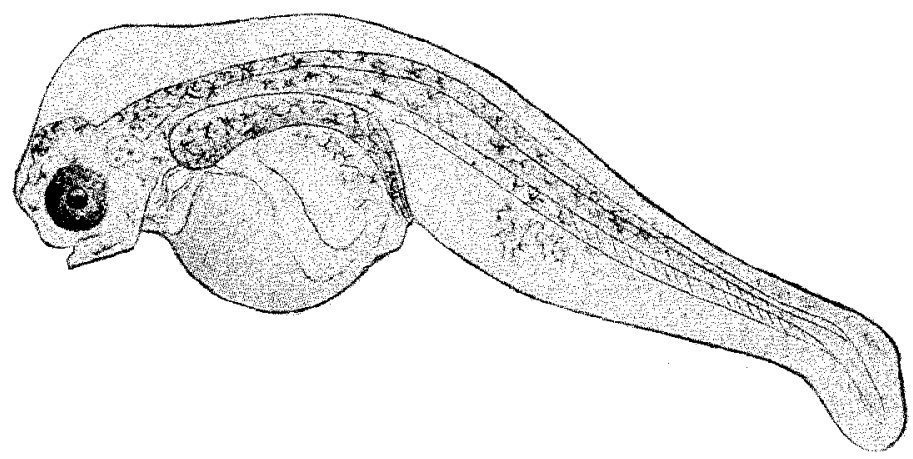

Abb. 31. Flunderlarve am 14. Tag nach Befruchtung des Eies, aus verdünntem Nordseewasser von $7,3 \% \%$.

Die nach dem 12. Versuchstage noch nicht geschlüpften Embryonen gingen zugrunde. Die Zeit des Schlüpfens in den geringen Salzgehaltsstufen zog sich also viel mehr in die Länge als im normalen Salzgehalt.

Dieselben Schädigungen, wie sie im Klieschenversuch im verringerten Salzgehalt auftraten, wurden auch an Flundereiern beobachtet. Aber in mehrfacher Hinsicht ist diese Wirkung geringer gewesen:

1. Die Quellung des Dotlérs trat bei Flundereiern erst am 4. Entwicklungstage auf; das ist eine Verzögerung gegenüber dem Klieschenversuch, bei dem sie schon am 2. Tage 
beobachtet wurde, um 2 Tage. Allerdings entwickelten sich die Flundereier gerade am Anfang des Versuchs wegen der etwas geringeren Temperatur $\left(4-7^{\circ} \mathrm{C}\right.$.) langsamer.

2. Erst die Salzgehaltsstufe von $11 \%$ rief an Flundereiern schädliche Wirkungen hervor, während Schädigungen an Kliescheneiern schon in $15 \%$, andeutungsweise sogar schon in $18 \%$ beobachtet wurden.

3. Auch in $7 \%$ gelangte ein Teil der Flunderembryonen zum Schlüpren. Klieschenembryonen, die während ihrer ganzen Eientwicklung dem Salzgehalt von $8 \%$ ausgesetzt waren, kamen dagegen nicht zum Schlüpfen.

4. Die Verzögerung der Schlüpfzeit dauerte im Klieschenversuch wesentlich länger als im Flunderversuch. Im Kliesehenversuch schlüpften am 1. Tag des Schlüpfens im normalen Salzgehalt in den Salzgehalten von 15-8\% noch keine Larven. Im Flunderversuch schlüpften am 1 . Schlüpftag im normalen Salzgehalt vereinzelt auch in allen anderen Salzgehaltsstufen einige Tiere. Die Schlüpfzeit in den geringen Salzgehaltsstufen zog sich bei Klieschen 6 Tage, bei Flundern nur 4 Tage hin.

Es fragt sich nun, ob für die günstigeren Ergebnisse im Flunderversuch die besseren Wasserverhältnisse verantwortlich gemacht werden müssen - im Klieschenversuch können ja auch andere Wasserverhältnisse als der Salzgehalt schädlich eingewirkt haben (s. S. 229) - oder ob sich in diesen physiologischen Unterschieden Artunterschiede zwischen Kliesche und Flunder bemerkbar machen. Es ist möglich, daß auch die Eier und Larven der Flunder, ähnlich wie die ausgewachsenen Tiere (Henschel 1936), gegen Salzgehaltsverdünnungen widerstandsfähiger sind als die anderer mariner Fische, wie etwa auch die der Kliesche. Diese Frage müßten weitere Versuche klären.

d) Besprechung der Ergebnisse: Di e Brackwasserschädigung der Entwicklung.

Als auffallendstes Merkmal der Brackwasserschädigungen trat die Quellung des Dotters in Erscheinung. Erst im Laufe der späteren Entwicklung wurde auch der Embryo selbst von starken Schädigungen, Verkrümmungen des Rumpfes und Verkümmerungen der Flossensäume betroffen. Es fragt sich, ob hierfür nicht lediglich der mechanische Druck, mit dem der gequollene Dotter den Embryo an die Eihaut preßt, verantwortlich gemacht werden muß. Sicher spielt auch dieser Faktor eine Rolle. Aber auf die Annahme direkter osmotischer Schädigungen der Embryogewebe wird man trotzdem nicht verzichten dürfen. Man darf nämlich garnicht erwarten, daß sich osmotische Schäden im Gewebe ebenso deutlich bemerkbar machen wie im Dotter; der Dotter besitzt wohl eine viel größere Quellungsfähigkeit als die Gewebszelle. Die Fortsetzung und Steigerung der Entwicklungsstörungen nun im Laufe der freien Larvenentwicklung liefert den Beweis, daß auch die Gewebe von osmotischen Schädigungen betroffen sein müssen. Ebenso sprechen die mehrfach (Seite 233 u. 234) erwähnten, unscharfen Umrisse der Brackwasserembryonen dafür. Sie machen den Eindruck, als ob auch die Gewebezellen gewisse Quellungen erlitten haben. Histologische Studien der Gewebe, ähnlich wie PALMherT (1933) dies an Hydroidpolypen ausgeführt hat, müBten diese Fragen beantworten. Als eine Wirkung des Brackwassers ist auch die Verlangsamung der Entwicklung aufzufassen. Diese müßte sich nun auch in einer Verringerung der Sauerstoffzehrung bemerkbar machen. Hierüber liegen auch einige Versuchsreihen vor, über die nun berichtet werden soll.

\section{Sauerstoffzehrung von Kliescheneiern im Seewasser verschiedenen Salzgehalts.}

\section{a) Versuchsanordnung.}

Die genaue Messung des Sauerstoffgehalts vor und nach Zehrung durch die Eier erforderte eine etwas komplizierte Versuchsanordnung ${ }^{1}$ ). Abb. 32 zeigt sie. Seewasser der gewünschten Konzentration wurde in eine Glasflasche Gl gefüllt und durchlüftet, bis Sauerstoffsättigung eingetreten war. Das sauerstoffgesättigte Wasser wurde durch eine Rohrleitung herausgehebert und durch einen Zylinder geschickt, der gegen das Kühlwasser, in dem er hing, abgedichtet war. In diesem (VR $=$ Versuchsröhre) befanden sich die Eier. Die Versuchsröhre hing, wie gesagt, in einem Becken mit Kühlwasser. Das Wasser, das zur Sauerstoffzehrung benutzt werden sollte, wurde vor Durchfließen der Versuchsröhre durch eine Kühlschlange (KS) geschickt, die ebenfalls im Kühlwasserbecken auf-

1) Vergl. Vorbemerkung S. $226 \mathrm{u}, 227$. 


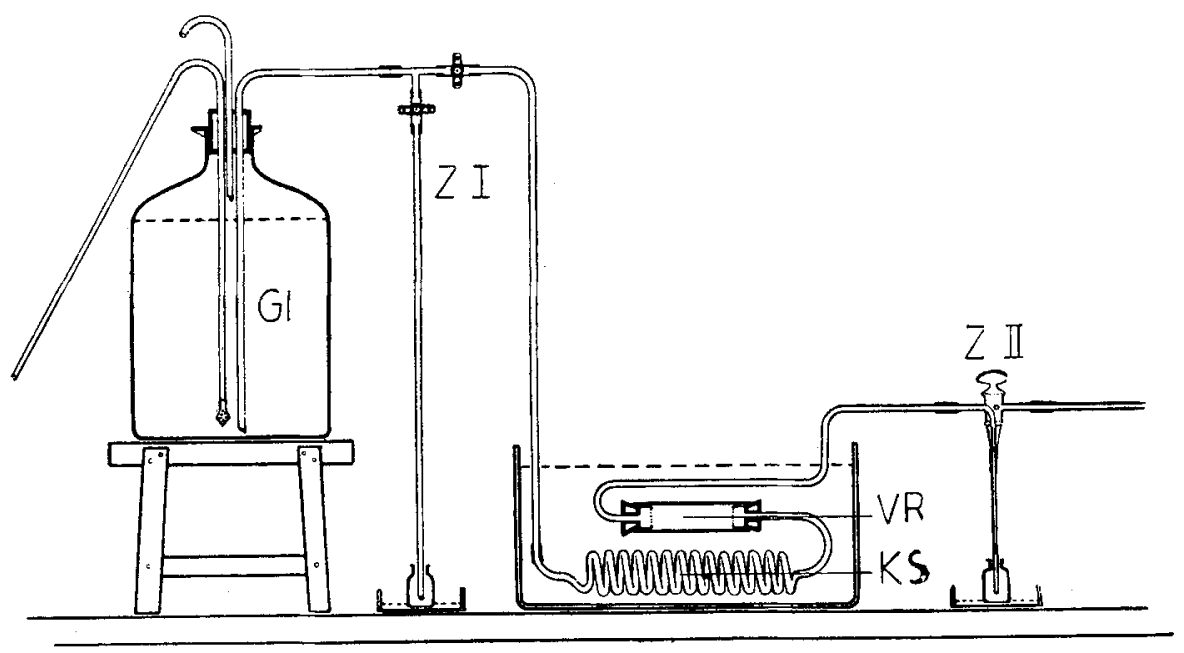

Abb. 32.

gehängt war. Es sollte sich der Temperatur des Kühlwassers bereits angeglichen haben, ehe es in die Versuchsröhre gelangte.

Vor und nach Sauerstoffzehrung, das heißt also vor und nach Passieren der Versuchsröhre, mußte je eine Wasserprobe zur Sauerstoffbestimmung entnommen werden. Hierfür dienten die beiden Zapfstellen Z I und Z II. Die Abbildung deutet die Handhabe dieser Entnahme an. Der Wasserzufluß durch die Versuchsröhre war so reguliert, daß in jeder Stunde drei Liter Wasser an den Eiern vorbeiflossen. Nach jeder Stunde wurden an den beiden Zapfstellen Wasserproben entnommen und der Sauerstoff vor und nach Passieren gemessen. Aus mehreren hintereinander ausgeführten Versuchen wurde das Mittel der Sauerstoffzehrung für jeden Versuchstag und für jede Salzgehaltsstufe errechnet. Es mußten für jeden Versuch gleiche Eimengen verwandt werden. Sie wurden mit einer Ballpipette abgemessen.

b) Versuchsergebnisse.

Als Beispiel für den Verlauf eines Versuchs geben wir das Protokoll des zweiten Tages der Eiserie auf $18,2 \%{ }^{\circ}$.

Protokoll.

Sauerstoffzehrung.

Versuchstier: Pleuronectes limanda, Eier.

Datum: 30. März 1935.

Eier in verdünntem Seewasser.

Temperatur: $8,9^{\circ} \mathrm{C}$.

Cl-Gehalt: $10,05 \%$.

Salzgehalt: $18,17 \%$.

14.0 Uhr 2 Proben entnommen: 1. vor den Eiern

2. hinter den Eiern.

An den Eiern wurde keine Teilung bemerkt.

Sauerstoffwerte :

cc Sauerstoff in 1 Liter

1. Probe: $\quad 7,190$

2. Probe: $\quad 7,140$

Differenz: $\quad 0,050$

\begin{tabular}{c} 
Sättigung \\
$97,55 \%$ \\
$97,08 \%$ \\
\hline $0,47 \%$
\end{tabular}

Es wird also die Sauerstoffsättigung vor und nach Passieren der Versuchsröhre gemessen. Die Zehrung, die Differenz zwischen den beiden Werten wird ausgedrückt in $\%$ der Sättigung. Zahlentafel 1 gibt das Gesamtergebnis wieder. 
Zahlentafel 1.

Sauerstoffzehrung von Kliescheneiern in $\%$ der Sättigung. (Temperatur $8-9^{\circ} \mathrm{C.}$ )

\begin{tabular}{c|c|c|c|c|c}
\hline $\begin{array}{c}\text { Tag nach } \\
\text { Befruchtung }\end{array}$ & 33,3 & $\mathbf{1 8 , 2}$ & 13,8 & 11,3 & 7,3 \\
\hline & & & & \multicolumn{5}{|c}{ Salzgehalt } \\
\hline. & 4,36 & 2,21 & 0,59 & 0,60 & 0,73 \\
3. & 1,21 & 0,47 & 1,25 & 1,44 & 1,08 \\
4. & 3,14 & 0,54 & 1,04 & 1,15 & 1,05 \\
5. & 3,23 & 2,83 & 1,11 & 0,51 & 2,26 \\
6. & 2,03 & 3,18 & 2,01 & 1,46 & - \\
7. & 1,78 & 1,54 & 2,35 & 3,80 & 1,49 \\
8. & 2,80 & $(6,65 ?)$ & 2,63 & 1,79 & 1,11 \\
9. & 1,67 & 1,44 & 0,81 & 2,94 & 0,83 \\
10. & 2,49 & 2,57 & 0,66 & - & 1,35 \\
\hline Mittel : & 1,59 & $(15,52 ?)$ & - & - & - \\
\hline Minimumwerte: & 2,43 & $\left.1,599^{1}\right)$ & 1,58 & 1,70 & 1,30 \\
& 1,21 & 0,47 & 0,59 & 0,51 & 0,73
\end{tabular}

Die Schwankungen sind groß. Die Mittelwerte liegen für die niedrigen Salzgehalte niedriger als für das normale Seewasser. Zahlenstatistisch gesichert sind diese Unterschiede allerdings nicht. Das ist bei der großen Schwankung der Einzelwerte und der geringen Anzahl der Versuche nicht verwunderlich. Trotzdem - muß man doch annehmen - erlauben die Zahlenreihen gewisse Rückschlüsse auf die Sauerstoffzehrung der Eier in diesen verschiedenen Salzgehaltsstufen. Versuchsfehler werden sich wohl hauptsächlich durch Absterben der Eier und damit verbundenem Bakterienwachstum oder durch Entwicklung von Ciliaten ergeben, wie sie in Eierzuchten fast stets beobachtet werden. Auch bei dauernd fließendem Wasser, wie es in der Versuchsanordnung vorlag, ist dies der Fall, weil die Organismen sich auf den Eihäuten anzusiedeln pflegen. Das heißt, die Versuchsfehler werden die Zehrungswerte einseitig abändern, sie werden sie immer eher erhöhen als erniedrigen. So werden z. B. die Werte 6,65 und 15,52\% Sättigungszehrung, die ja vollständig aus dem Rahmen fallen, auf solche Versuchsfehler zurückzuführen sein. Man wird also annehmen können, daß die kleinsten Werte dem wahren Wert am nächsten kommen. Vergleicht man nun diese kleinsten Zehrungswerte der verschiedenen Salzgehalte miteinander, fallen die niedrigeren Werte der geringen Salzgehaltsstufen doch ganz anders ins Gewicht. Denn auch dies ist zu beachten: Die Fehler werden in den geringen Salzgehalten größer sein, weil ja die Sterblichkeit der Eier dort höher ist, die „Verpilzung“ also größer sein wird. Trotzdem wurden so niedrige Zehrungswerte wie in den Verdünnungstufen in normalem Salzgehalt nie gefunden, ein Hinweis darauf, daß die Sauerstoffzehrung der Kliescheneier im Brackwasser geringer ist als in normalem Seewasser. Diese Beobachtung deckt sich auch mit den im vorigen Abschnitt besprochenen Versuchsergebnissen, daß sich die Entwicklung von Kliescheneiern in verdünntem Seewasser verlangsamt.

Dies Ergebnis deckt sich ebenfalls mit Beobachtungen von LEINER (1936 Seite 182 ff. und 1938 Seite 53 ff.), die er an Embryonen des Seepferdchens und einiger Seenadeln machte. Der Sauerstoffverbrauch je Stunde und $\mathrm{mg}$ Trockengewicht nahm mit Salzgehaltsverdünnung $a b$, er erreichte bei einem Seewasser, das auf $1 / 2-1 / 4$ des normalen Salzgehaltes verdünnt war, ein Minimum, stieg bei noch geringeren Konzentrationen wieder an, ohne aber den Ausgangswert in normalem Salzgehalt zu erreichen.

Zwischen den einzelnen Verdünnungsstufen ergab sich aber kein Unterschied in der Sauerstoffzehrung. Hierfür mögen die Versuchsfehler zu groß gewesen sein. Die Versuche müßten wiederholt und das Ergebnis an den Eiern anderer Fischarten müßte nachgeprüft werden.

\section{Die Befruchtungsrate von Kliescheneiern im Seewasser verschiedenen Salzgehalts.}

Zunächst wurde die Beweglichkeit der Spermien in verschiedenen Salzgehaltsstufen geprüft. Benutzt wurde Sperma von Kliesche, Scholle und Flunder. Es wurde unter dem Mikroskop in den verschiedenen Verdünnungsstufen beobachtet. Die Beweglichkeit nahm in den niedrigeren Salzgehalten von $18 \%$ an stark ab. Die Spermatozoen waren in $15 \%$ nur noch schwach, in $13 \%$ überhaupt nicht mehr beweglich.

1) Die Werte 6,65 und 15,52 nicht mit berechnet. 
Für die Hauptversuche wurde das Salzgehaltsgefälle von 31,5 bis $7 \%$ in 15 Stufen unterteilt und die Befruchtungsrate der einzelnen Stufen durch Auszählen der gefurchten und der unentwickelten Eier ermittelt. Die Zählung fand statt, als die Mehrzahl der entwickelten Eier das 4-Zellenstadium erreicht hatte, das war etwa vier Stunden nach Befruchtung.

Es werden in der Zahlentafel 2, die das Ergebnis zusammenfaßt, verschiedene Rubriken aufgeführt. die einer Erläuterung bedürfen. Neben regelmäßigen Furchungsstadien fanden sich - zum Teil recht häufig - unregelmäßig gefurchte Eier. Beide Furchungsbilder sind getrennt aufgezählt. Die unregelmäßig Gefurchten wurden in Kontrollserien weiter beobachtet. Sie bildeten zum größten Teil Keimscheiben aus. Einige allerdings entwickelten sich nicht weiter. Unter ihnen können auch unbefruchtete Eier gewesen sein, die im Absterben begriffen waren. Denn solche Eier weisen oft Schrumpfungen auf, die unregelmäßige Furchungen vortäuschen. Weiter unterscheidet die Zahlentafel lebende, unentwickelte Eier und solche, die zur Zeit der Furchungsstadien schon abgestorben waren, ohne selbst Furchungszellen gebildet zu haben. Es geht aus dem Bericht nicht hervor, wie weit eine Gewähr dafür gegeben werden kann, daß die Abgestorbenen unbefruchtet waren. Es ist aber anzunehmen, daß sie es waren. Denn selbst von den lebenden, ungefurchten Eiern ging der größte Teil zugrunde, ohne Furchungszellen abgeschnürt zu haben.

\section{Zahlentafel 2.}

Hundertsätze der Eientwicklung nach der Befruchtung in Seewasser mit verschiedenem Salzgehalt.

\begin{tabular}{|c|c|c|c|c|c|c|}
\hline \multirow[b]{2}{*}{$\begin{array}{l}\text { Salz- } \\
\text { gehalt }\end{array}$} & \multicolumn{6}{|c|}{ Hundertsätze der Entwicklungsstidien } \\
\hline & $\begin{array}{l}\text { regelmäßig } \\
\text { gefurcht }\end{array}$ & $\begin{array}{l}\text { unregel- } \\
\text { mäßig } \\
\text { gefurcht }\end{array}$ & $\mid \begin{array}{c}\text { Summe der } \\
\text { gefurchten } \\
\text { Eier }\end{array}$ & $\begin{array}{c}\text { unent- } \\
\text { wickelt } \\
\text { klare Eier }\end{array}$ & $\begin{array}{c}\text { unent- } \\
\text { wickelt } \\
\text { tote Eier }\end{array}$ & $\begin{array}{l}\text { Zahl der } \\
\text { Beob- } \\
\text { achtungen }\end{array}$ \\
\hline $31, \overline{0} 0$ & 86 & 5 & 91 & - & 9 & 280 \\
\hline 30,07 & 45 & 32 & 78 & 5 & 18 & 266 \\
\hline 28,20 & - & 68 & 68 & 5 & 27 & 261 \\
\hline 25,82 & - & 62 & 62 & 8 & 30 & 292 \\
\hline 24,15 & 19 & 65 & 84 & 7 & 9 & 243 \\
\hline 22,24 & 16 & 56 & 72 & 9 & 19 & 245 \\
\hline 20,43 & 8 & $6 \bar{a}$ & 73 & 8 & 19 & 275 \\
\hline 18,66 & - & 42 & 42 & 30 & 28 & 243 \\
\hline 16.80 & - & 26 & 26 & 45 & 29 & 262 \\
\hline 15,02 & - & - & - & 61 & 39 & 280 \\
\hline 13,22 & 一 & - & - & 48 & 52 & 310 \\
\hline 11,41 & - & - & - & 38 & 62 & 300 \\
\hline 10,00 & - & - & 一 & 30 & 70 & 300 \\
\hline 8,23 & - & - & - & - & 100 & 265 \\
\hline 7,91 & - & - & $\cdots$ & - & 100 & 273 \\
\hline
\end{tabular}

Befruchtungen fanden nach den Ergebnissen statt bis zu der Salzgehaltsstufe von $16,8 \%$, doch nimmt die Befruchtungsrate bereits in $18 \%$ stark ab. Von $32-20 \%$ zeigen die Werte dagegen keine Kongruenz zur Salzgehaltsverringerung. Sie sind in 28 und $25 \%$ relativ niedrig, in 24 und $22 \%$ wieder höher. In den Salzgehalten von 28 und $25 \%$ wurden keine regelmäßig gefurchten Eier gefunden. Auch war die Zahl bereits abgestorbener Eier hier relativ hoch. Es müssen hier Versuchsfehler vorgelegen haben. Vielleicht ist Sperma und Eimaterial nicht gleichwertig gewesen, etwa so, daß in die mittleren Salzgehaltsstufen nicht vollständig ausgereifte Fortpflanzungsprodukte gelangt sind. In $15 \%$ wurde eine deutliche Abnahme der Spermienbeweglichkeit beobachtet. In diesen Salzgehaltsstufen kamen auch keine Befruchtungen mehr zustande. Man wird daher das Sperma für den Ausfall der Befruchtung in niederen Salzgehalten verantwortlich machen können. Aber die größere Zahl unregelmäßig gefurchter Eier in den mäßig erniedrigten Salzgehaltsstufen und das schnellere Absterben unbefruchteter Eier darin zeigt an, daß auch das Ei der Kliesche, wenn es zur Befruchtung in niedrigen Salzgehalt überführt wird, Schädigungen erleidet, die an dem schlechten Ausfall der Befruchtungsrate Anteil haben werden. 


\section{Vergleich zwischen den Versuchsergebnissen und den Beobachtungen an Eiern und Larven in der freien Ostsee.}

Die Arbeit ging aus von der Frage, welchen Einfluß der Salzgehalt eines Brackwassergebiets, etwa der Ostsee, oder der Einflußzone einer Flußmündung auf die Entwicklung mariner Fischeier ausüben mag. Die Lösung dieses Problems ist durch die Versuchsergebnisse gefördert worden. Ei und Sperma, in niedrigem Salzgehalt abgelegt, erleiden Schädigungen, die die Befruchtungsrate vermindern, schließlich bei genügend starker Verdünnung des Seewassers eine Befruchtung überhaupt nicht mehr zustande kommen lassen. Die befruchteten Eier erleiden im Laufe der Entwicklung weitere Schädigungen, Dotterquellungen, Entwicklungsstörungen, die zum Teil Krüppelformen hervorrufen. Embryo oder Larve gehen schließlich zugrunde.

Bei den lebensfähig gebliebenen Eiern und Larven tritt der Einfluß des niedrigen Salzgehalts abgeschwächt in einer Verlangsamung der Entwicklung wieder in Erscheinung. Hiermit offenbar im Zusammenhang konnte eine Verminderung der Sauerstoffzehrung in verdünntem Salzgehalt festgestellt werden. Die kritische Salzkonzentration, von welcher an Schädigungen überhaupt beobachtet wurden, ist $18 \%$. Es ist dieselbe Konzentration, die auch für ausgewachsene Tiere einen Wendepunkt bedeutet. Von dieser Stufe an besteht auch für diese Gefahr einer zusätzlichen Wasseraufnahme in den Geweben. Dieser kritische Salzgehaltswert hat die Isotonie zwischen Außenmedien und interzellulärer Flüssigkeit zwar noch nicht ganz erreicht, liegt ihr aber nahe.

Befruchtungen von Kliescheneiern kamen bereits in dem Salzgehalt 15\% nicht mehr zustande. Entwicklungsstörungen von Eiern, die im normalen Seewasser befruchtet waren, traten auch deutlich zuerst in dieser Salzgehaltsstufe in Erscheinung. Für die Flunder liegen die Verhältnisse etwas günstiger. Schädigungen haben sich bei ihr erst in 11 und $7 \%$ Salzgehalt gezeigt. In der Einleitung wurde über Beobachtungen toter oder nur schlecht lebensfähiger Fischeier in Planktonfängen der Ostsee gesprochen. Diese Erscheinungen finden nun in den schädigenden Einflüssen des niedrigen Salzgehalts während der Befruchtung und der Embryonalentwicklung eine befriedigende Erklärung.

Auch die Weiterentwicklung der Larven wurde in verdünntem Nordseewasser gestört. Hier zeigen sich wieder Parallelen zwischen den experimentellen Ergebnissen und gewissen Beobachtungen in freier Natur. Auf Seite 227 u. 228 wurde über Verzögerungen in der Differenzierung der Organe von Ostseefischen gegenüber den entsprechenden Arten aus der Nordsee berichtet. Im Experiment wurde ebenfalls eine Verlangsamung der Entwicklung derjenigen Larven beobachtet, die in Seewasser verringerten Salzgehalts gehalten wurden. Dies spricht sehr für eine Auffassung, welche die verspätete Differenzierung der Gewebe auf Salzgehaltseinflüsse, nämlich auf eine Verlangsamung der Entwicklungsvorgänge im brackischen Seewasser zurückführen möchte.

Es läßt sich aber nicht leugnen, daß Sperma, Ei und Larve von Seewasserfischen in der Ostsee auch Anpassungen an den geringen Salzgehalt des Meerwassers erworben haben müssen. Die Kliesche zum Beispiel laicht in der Ostsee in einem Salzgehalt, in dem im vorliegenden Versuch nur noch geringe oder überhaupt keine Befruchtungen mehr beobachtet wurden. Ein 'Hauptlaichgebiet dieser Art ist z. B. das Bornholmbecken. (MIELCK und KüNNE zit. S. 48.) Der Salzgehalt der Bodenschicht beträgt in diesem Gebiet 15-17\% (Schulz 1932 und 1935, WatTenberg 1940). Die Befruchtungsrate nahm ja im Versuch von $18 \%$ an stark ab, in $15 \%$ wurden überhaupt keine Befruchtungen mehr erzielt. Aber in freier Natur wurden sogar noch in der Gotlandmulde befruchtete Eier gefischt (MIELCK und KünNe zit.) in einem Gebiet, dessen Salzgehalt in der Bodenschicht weniger als $12 \%$ beträgt (Schulz, WatTEnBERG zit.).

Für die Flunder, die im Bornholmbecken laicht; ist der Salzgehalt von 15-17\% auch nach den Versuchsergebnissen noch durchaus erträglich. Die Schädigungen setzten bei dieser Art erst in einem Salzgehalt von $11 \% / n$ ein. Allerdings wurden Befruchtungsversuche in den verschiedenen Salzgehaltsstufen nicht ausgeführt. Es gibt aber in der Ostsee außer der Flunder, die zur Laichzeit in die tiefen Becken mit ihren salzreichen Bodenwasserschichten abwandert (daher als "Tiefenflunder" bezeichnet), noch eine andere physiologische Rasse, wie Strodtmann und KändLER (KäNDLer 1934, StrodtMann und KändLer 1935) nachwiesen, die sogenannte "Bankflunder“. Diese Flunder sucht zur Laichzeit die 
flachen Bänke der mittleren Ostsee, besonders die Oder- und Stolpebank auf. Sie besitzt kleinere Eier als die Tiefenflunder, die während der Entwicklung nicht schweben, sondern am Boden liegen. Ihre Entwicklung findet in der Oberflächenschicht des Ostseewassers statt, also in einem Salzgehalt von $7-8 \%$. In diesen Salzgehaltsstúfen war aber die Nordseeflunder bereits von starken Schädigungen betroffen.

Allerdings muß einschränkend zu diesen Ausführungen hervorgehoben werden, daß verdünntes Nordseewrasser nicht ohne weiteres mit Ostseewasser verglichen werden darf. So weist Schulz (1932, I d, S. 61) auf die positive Anomalie der Alkalinität der gesamten mittleren Ostsee hin, die zurückzuführen ist auf einen hohen Kalziumbicarbonatgehalt.' Die Bedeutung gerade des Kalziums für die Eientwicklung hat HERBST (1904) am Seeigelkeim nachgewiesen. Aber gerade in den salzreichen Tiefenschichten, in denen das Laichen hauptsächlich stattfindet, wird diese günstige Eigenschaft durch eine andere ungünstige Eigenschaft wahrscheinlich wieder abgeschwächt, nämlich durch den hohen Kohlensäuregehalt der Bodenschicht (Schulz 1932). Diese verschiedenen chemischen Eigenschaften des Wassers, kombiniert mit Salzgehaltsunterschieden, könnten im Experiment erst endgültig darüber Auskunft geben, in wieweit die Fische der Ostsee Anpassungen an das veränderte Milieu dieses Binnenmeeres erfahren haben.

\section{Zusammenfassung.}

1. Künstlich befruchtete Kliescheneier wurden in verschieden stark verdünnte Salzgehaltsstufen überführt, sofort nach Befruchtung oder einige Tage nach Einsetzen der Entwicklung. Es zeigten sich typische Schädigungen in den Salzgehalten von $15 \%$ und niedriger: Quellungen des Dotters, Verlangsamung der Entwicklung gegenüber Tieren im normalen Salzgehalt und pathologische Bildungen am Embryo. In der niedrigsten Salzgehaltsstufer von $8 \%$ gingen in einer Zucht alle, in einer anderen Zucht die meisten Larven zug unde. Die Schädigungen waren geringer, je später die Tiere in die verdünnten Salzgehalte gesetzt wurden. Auch nach dem Schlüpfen setzten sich die Entwicklungsstörungen in den niedrigen Salzgehalten fort.

2. Ähnliche Versuche wurden mit Flundereiern unternommen. Die Versuchsanordnung dieser Experimente wurde verbessert, derart, daß die Eier dauernd in fließendem Wasser gehalten werden konnten. Die Schädigungen waren bei diesen Versuchen viel geringer, wenn sie auch dieselben typischen Merkmale zeigten. So begannen die ersten Schädigungen sich erst im Salzgehalt von 11\% bemerkbar zu machen. In $8 \%$ dagegen kamen noch reichlich Larven zum Schlüpfen. Nach den Versuchen kann nicht entschieden werden, ob die besseren Ergebnisse auf den Einfluß der besseren Wasserverhältnisse zurückzuführen sind, oder auf Artunterschieden zwischen Flunder und Kliesche beruhen. Man muß vermuten, daß Artunterschiede auch hierin bestehen, da ja die Flunder in verschiedener Hinsicht an das Leben im Brackwasser angepaßt ist. Sodann wurde die Sauerstoffzehrung von Kliescheneiern in verschiedenem Salzgehalt gemessen. Wenn die Ergebnisse auch keine zahlenstatistisch gesicherten Unterschiede gezeitigt haben, so liegen die Minimalwerte der Sauerstoffzehrung in den niedrigen Salzgehaltsstufen auffallend niedriger als im normalen Seewasser. Die Versuchsfehler müßten aber in den niedrigen Salzgehaltsstufen die Zehrungswerte nach der zu hohen Seite verschieben (stärkere Verpilzung der Eier im niedrigen Salzgehalt) Man kann also annehmen, daß die Sauerstoffzehrung im Seewasser geringen Salzgehalts geringer ist, was mit der Beobachtung der Wachstumsverzögerung der Eier gut in Einklang zu bringen ist.

3. Befruchtungsversuche im normalen und Seewasser von verringertem Salzgehalt wurden ebenfalls an Kliescheneiern vorgenommen. Sie ergaben, daß im Salzgehalt von $15 \%$ an keine Befruchtungen mehr zustande kommen, stärkere Ausfälle gibt es aber schon in $18 \%$.

4. Am Schluß wurde bemerkt, daß die Ergebnisse die schlechten Entwicklungsverhältnisse mariner Fischeier und Larven in brackischen Meeresgebieten, z. B. der Ostsee, erklären. Man muß aber feststellen, daß die Ostseeformen auch Anpassungen an die schlechten Salzverhältnisse erworben haben. 


\section{Schriftenverzeichnis.}

APSTEIN, C. Die Verbreitung der pelagischen Fischeier und Larven in der Beltsee und den angrenzenden Meeresteilen 1908/09. Wiss. Meeresunt. N. F. Abt. Kiel, 13, 1911, S. 225-284.

BEADLE, S. C., The effect of salinity changes on the water content and respiration of marine vertebrates. Journ. of exp. biol. Cambridge, 8, 1931, S. 211-227.

Henschel, J., Wasserhaushalt und Osmoregulation von Scholle und Flunder. Wiss. Meeresunt. N. F. Abt. Kiel, 22. 1936 , S. $89-121$.

HERBST, C., Ueber die zur Entwicklung der Seeigellarven notwendigen organischen Stoffe, ihre Rolle und Vertretbarkeit. Arch. f. Entwicklungsmech. 17, 1904, S. 306-520.

KäNdLer, R., Rapp. et Proc. Verb. Kopenhagen, 89, 1934, S. 57.

LEINER, M, Die physiologischen Grundlagen der Nachkommenfürsorge bei den Seenadeln (Syngnathidae). Z. Vergl. Physiol. 23, 1936, S. 147-207.

-, Die Atmung des kurzschnäuzigen Seepferdchens (Hippocampus brevirostris Cuv.) und seiner Embryonen und Jungfische. Z. Vergl, Physiol. 24, 1937, S. 143-166.

-, Die Physiologie der Fischatmung. Leipzig 1938.

MELCK, W, und CL. KÜNNE, Fischbrut und Planktonuntersuchungen auf dem Reichsforschungsdampfer „Poseidon" in der Ostsee Mai-Juni 1931. Wiss. Meeresunt. N. F. Abt. Helgoland 19, Nr. 7, 1935, S. $4-120$.

Palmert, H. W., Beiträge zum Problem der Osmoregulation einiger Hydroidpolypen. Zool. Jahrb. Physiol. 52, 1933 , S. $212-260$.

REIBISCH, J., Bericht der preußischen Kommission zur wissenschaftlichen Untersuchung der deutschen Meere in Kiel über ihre Tätigkeit im Rechnungsjahr 1930. Mitt. d. deutsch. Seefischereivereins, Berlin, 47, 1931, S. 349-351.

Remane, A., Die Brackwasserfauna. Verhandl. deutsch. zool. Ges. 7, Supplementband, d. Zool. Anz. 1934, S. $34-74$.

SCHLIEPER, C., Die Osmoregulation wasserlebender Tiere. Biol. Rev. Cambridge, 5, 1930, S. $309-356$.

ScHurz, B., Die hydrographischen Arbeiten der Deutschen Wissenschaftlichen Kommission für Meeresforschung 1930-1933. IV. Arbeiten der Ostsee. Ber. d. deutsch. wissensch. Komm. f. Meeresf. N. F., 7, 1935 , S. $203--209$.

- Einführung in die Hydrographie der Nord- und Ostsee. In Grimpe-Waglers Tierwelt der Nord- und Ostsee, 1932 , I d. S. $4 \overline{5}-88$.

StrodtmanN, S., Laichen und Wandern der Ostseefische. 2. Bericht. Wiss. Meeresunt. N. F. Abt. Helgoland, 7, 1906 , S. $132-216$.

-, Weitere Untersuchungen über Ostseefische. Wiss. Meeresunt. N. F. Abt. Helgoland, 14, 1918, S. 29-95.

STRODTMANN, S: und R. KÄNDLER, Die Arbeiten der Ostseeabteilung Hamturg in den Jahren 1930-1933. Ber. d. deutsch. wiss. Komm. f. Meeresf. N. F. 7, 1935, S. 269-318.

Thienemann, A., Mysis relicta im sauerstoffarmen Tiefenwasser der Ostsee und das Problem der Atmung in Salzwasser und Süßwasser. Zool. Jahrb. Physiol. 45, 1928, S. 371-384.

Wattenberg, H., Der hydrographisch-chemische Zustand der Ostsee im Sommer 1939. Ann. d. Hydr. u. mar. Meteor. 68, 1940, S. $185-194$. 